\title{
FDTD Analysis and Measurement of Aperture Antennas Based on the Triplate Transmission-Line Structure
}

\author{
Hisamatsu Nakano, Fellow, IEEE, Tomohiro Unno, Kazuo Nakayama, Member, IEEE, \\ and Junji Yamauchi, Member, IEEE
}

\begin{abstract}
First, two aperture antennas designated as $P$-type and $C$-type antennas are analyzed using the finite-difference timedomain (FDTD) method. Each antenna is made of a triplate transmission line (TTL). A square aperture is cut out of the top plate of the TTL for both antennas to allow radiation. The bottom of the TTL is a planar plate for the $P$-type antenna and a plate with a hollow cavity for the $C$-type antenna. The power flow is expressed using Poynting vectors. It is revealed that parallel-plate mode power in the TTL is lower in the $C$ type antenna, compared with that in the $P$-type antenna. Second, an array antenna composed of two $C$-type elements is analyzed. The Poynting vector distribution in the aperture is found to remain almost unchanged when the element spacing is varied. The input impedance of the array antenna converges at an element spacing of approximately 0.9 wavelength. The theoretical radiation patterns are in good agreement with measured data.
\end{abstract}

Index Terms - Aperture antenna, FDTD analysis, triplate transmission line.

\section{INTRODUCTION}

A conventional aperture antenna is a flat radiation element made of a triplate transmission line (TTL), as shown in Fig. 1(a) [1]. The input power is converted to radiated power through the aperture cut in the top plate of the TTL. Usually, the conversion efficiency of input power to radiated power is not high due to generation of parallel-plate mode power, which propagates between the top and bottom plates of the TTL.

When designing aperture antennas based on the TTL structure, it is essential to suppress or reduce the parallel-plate mode power. One method of suppressing this power is found in [2], where shorting pins are used around a narrow aperture (slot) cut in the TTL. This technique is also applied to conductorbacked transmission lines [3].

This paper presents a novel aperture antenna with a cavity [C-type antenna shown in Fig. 1(b)] to reduce parallel-plate mode power. The structure of the $C$-type antenna is different from the waveguide aperture antenna with a suspended stripline (WA-SS) [4] and it is simpler and lighter than the WA-SS.

The $C$-type antenna is analyzed using the finite-difference time domain (FDTD) method [5]. The radiation characteris-

Manuscript received May 14, 1998.

The authors are with the Nakano Laboratory, College of Engineering, Hosei University, Koganei, Tokyo, 184-8584 Japan.

Publisher Item Identifier S 0018-926X(99)05805-6.

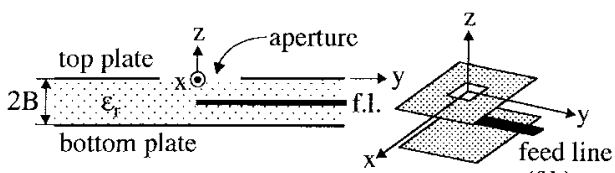

(f.l.)

(a)

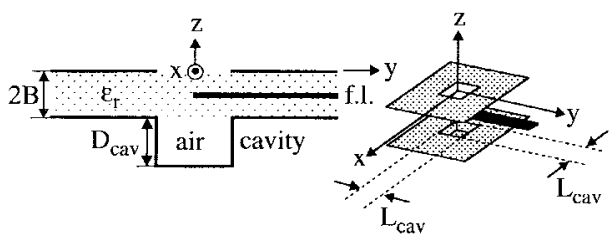

(b)

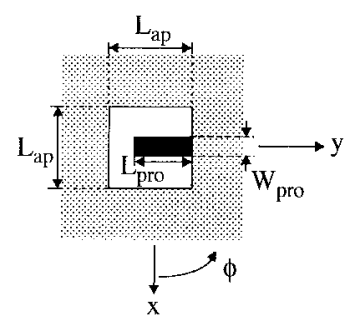

(c)

Fig. 1. Configuration of aperture antennas. (a) $P$-type antenna. (b) $C$-type antenna. (c) Top view (common to $P$ - and $C$-type antennas).

tics, including the input impedance, radiation pattern, radiation efficiency, and gain, are calculated. For comparison, the FDTD solutions to the conventional aperture antenna shown in Fig. 1(a) are also presented. It is revealed that the cavity shown in Fig. 1(b) is useful in reducing the effects of the parallel-plate mode power on the radiation characteristics.

Further investigation is performed for an array antenna composed of two $C$-type elements. The effects of the element spacing on the Poynting vector distributions in the apertures are investigated. The input impedance as a function of element spacing is also investigated. Some measured data are presented for checking the validity of the FDTD solutions.

\section{CONFiguration}

We investigate conventional and newly proposed aperture antennas whose cross-sectional views in the $y$ - $z$ plane are shown in Fig. 1(a) and (b), respectively. The antennas in Fig. 1(a) and (b) are designated as $P$ - and $C$-type antennas, 
respectively. The top view of these antennas is illustrated in Fig. 1(c).

Each antenna, made of a TTL, has a square aperture cut in the top conducting plate. The aperture of side length $L_{\text {ap }}$ is excited by a microstrip line probe located at $z=-B$. This microstrip line probe (length $L_{\text {pro }}$, width $W_{\text {pro, and }}$ thickness $T_{\text {pro }}$ ) is embedded in a dielectric material of relative permittivity $\varepsilon_{r}$.

The bottom of the TTL at $z=-2 B$ is made of a planar conducting plate for the $P$-type antenna, while the bottom for the $C$-type antenna is made of a conducting plate backed by a hollow cavity with depth $D_{\text {cav }}$. The side length of the cavity $L_{\text {cav }}$ is the same as that of the aperture in the top plate: $L_{\text {cav }}=L_{\text {ap }}$.

The following configuration parameters are held constant throughout this paper: $L_{\mathrm{ap}}=13 \mathrm{~mm}=0.514 \lambda_{0}, W_{\text {pro }}=$ $2 \mathrm{~mm}=0.079 \lambda_{0}, T_{\text {pro }}=0 \mathrm{~mm}, \varepsilon_{r}=1.2, B=2 \mathrm{~mm}=$ $0.079 \lambda_{0}$, and $D_{\text {cav }}=6.3 \mathrm{~mm}=0.249 \lambda_{0}$, where $\lambda_{0}$ is the free-space wavelength at a test frequency of $f=11.85 \mathrm{GHz}$ $\equiv f_{0}$. Only the probe length $L_{\text {pro }}$ is changed subject to the objectives of analysis.

The hollow cavity is regarded as a square waveguide terminated with a conducting plate. Note that the waveguide in this paper works at a frequency above the cutoff frequency of the $\mathrm{TE}_{10}$ mode. Discussions on the antenna characteristics below the cutoff frequency are found in [6].

\section{ANALYSIS METHOD AND RESUltS}

The FDTD method is adopted for analyzing the two aperture antennas shown in Fig. 1, where Yee's algorithm [7] is used with Liao's second-order absorbing boundary condition [8]. Each antenna configuration is symmetrical with respect to the $y$ - $z$ plane. Therefore, the analysis space can be reduced to onehalf of the full analysis space using a magnetic wall condition in the $y$ - $z$ plane (magnetic fields $H_{y}=0$ and $H_{z}=0$ in the $y$-z plane) [9].

The Poynting vector is calculated from $\mathbf{P}(\mathbf{R})=$ $\frac{1}{2} \operatorname{Real}\left[\mathbf{E}(\mathbf{R}) \times \mathbf{H}(\mathbf{R})^{*}\right]$, where $\mathbf{E}(\mathbf{R})$ and $\mathbf{H}(\mathbf{R})$ are, respectively, the electric and magnetic fields at an observation point specified by a position vector $\mathbf{R}$. The asterisk $\left(^{*}\right)$ designates a complex conjugate. The $\mathbf{E}(\mathbf{R})$ and $\mathbf{H}(\mathbf{R})$ are evaluated from time-domain fields $\mathbf{E}(\mathbf{R}, t)$ and $\mathbf{H}(\mathbf{R}, t)$, respectively [10].

The radiation field $\mathbf{E}_{\mathrm{far}}=E_{\theta} \hat{\theta}+E_{\phi} \hat{\phi}$ expressed in the spherical coordinates $(R, \theta, \phi)$ with unit vectors $(\hat{R}, \hat{\theta}, \hat{\phi})$ is calculated using the equivalence principle [11], [12]. The equivalent magnetic current density $\mathbf{M}_{\mathbf{a p}}\left(\mathbf{R}^{\prime}\right)$ over the aperture in the top plate specified by a position vector $\mathbf{R}^{\prime}$ is calculated from $\mathbf{E}_{\mathrm{ap}}\left(\mathbf{R}^{\prime}\right) \times \hat{n}$, where $\mathbf{E}_{\mathrm{ap}}\left(\mathbf{R}^{\prime}\right)$ is the electric field at the aperture and $\hat{n}$ is an outward unit vector normal to the aperture. The $E_{\theta}$ and $E_{\phi}$ are [12]

$$
\begin{aligned}
& E_{\theta}=\frac{j k_{0}}{4 \pi} \frac{e^{-j k_{0} R}}{R}(-\mathbf{L} \cdot \hat{\phi}) \\
& E_{\phi}=\frac{j k_{0}}{4 \pi} \frac{e^{-j k_{0} R}}{R}(\mathbf{L} \cdot \hat{\theta})
\end{aligned}
$$

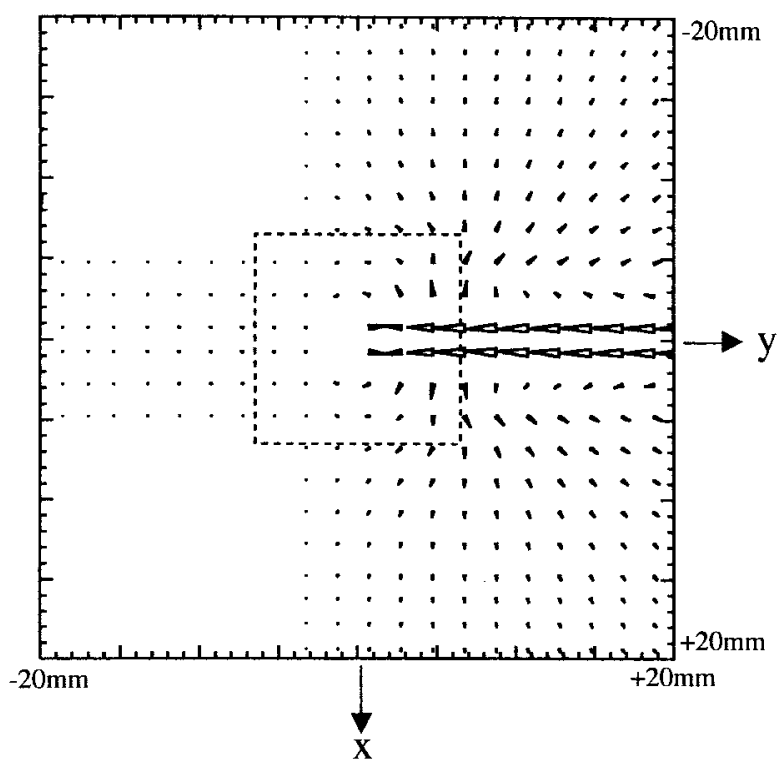

(a)

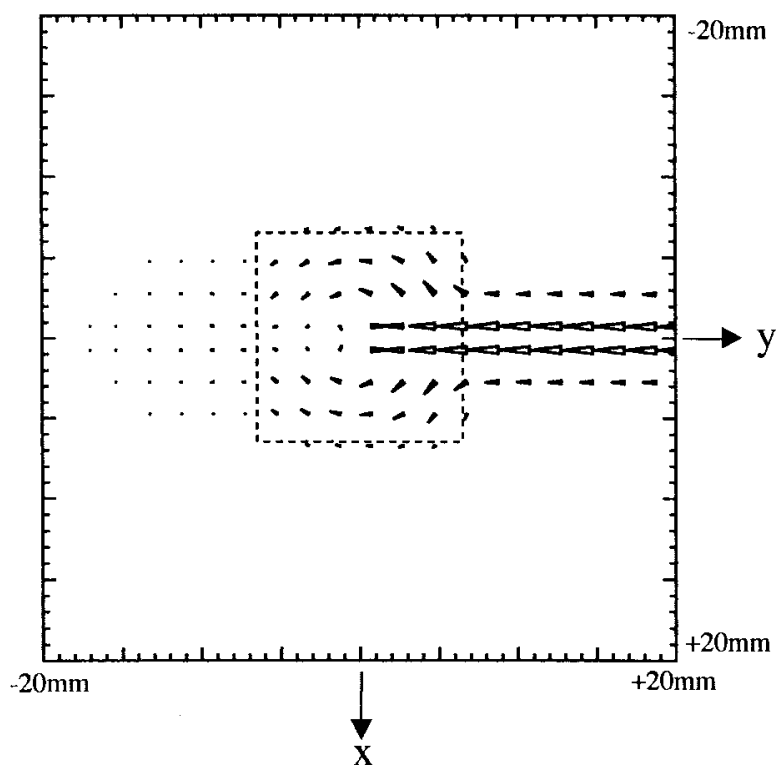

(b)

Fig. 2. Poynting vectors observed in a plane parallel to the $x-y$ plane at $z=-B+\Delta z / 2$, where $\Delta z=0.5 \mathrm{~mm}=0.02 \lambda_{0}$. (a) $P$-type antenna. (b) $C$-type antenna.

where $k_{0}\left(=2 \pi / \lambda_{0}\right)$ is the phase constant in free-space and

$$
\mathbf{L}=\int_{\text {aperture }} 2 \mathbf{M}_{\mathbf{a p}}\left(\mathbf{R}^{\prime}\right) e^{j k_{0} \hat{R} \cdot \mathbf{R}^{\prime}} d S^{\prime}
$$

The radiation efficiency $\eta$ is defined as the ratio of the power radiated from the aperture into free-space to the power input to the aperture $\mathbf{P}_{\text {in }}$

$$
\eta=\frac{\int_{\text {aperture }} \mathbf{P}\left(\mathbf{R}^{\prime}\right) \cdot \hat{n} d S^{\prime}}{\mathbf{P}_{\text {in }}} .
$$

\section{A. Poynting Vectors and Radiation Pattern}

We investigate the behavior of the parallel-plate mode power distributed between the top $(z=0)$ and bottom $(z=-2 B)$ plates. For this, the probe length is chosen to be one-half of the 


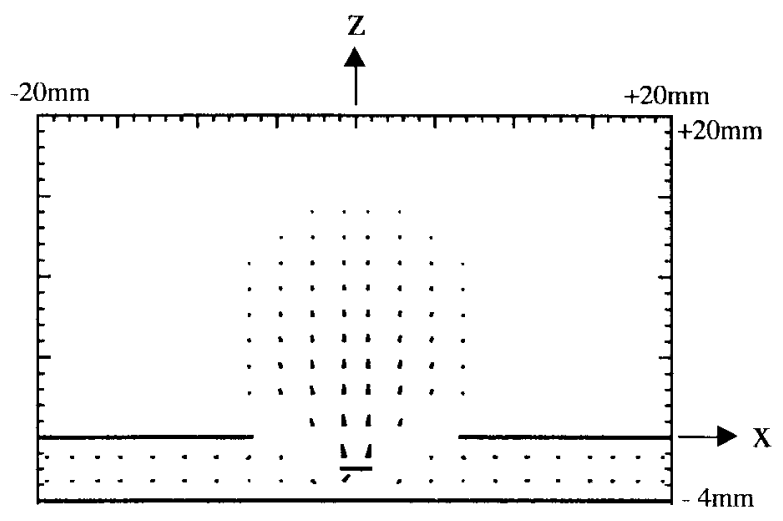

(a)

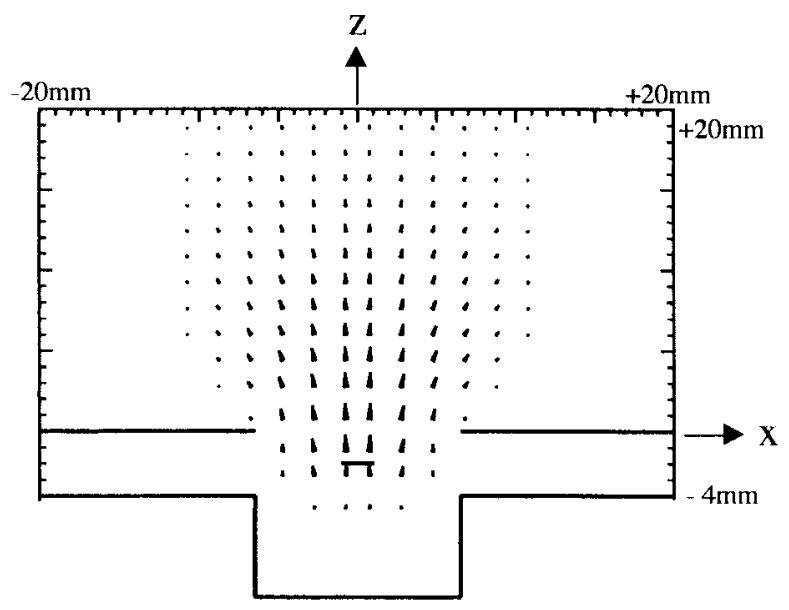

(b)

Fig. 3. Poynting vectors in the $x-z$ plane. (a) $P$-type antenna. (b) $C$-type antenna.

aperture side length: $L_{\text {pro }}=L_{\text {ap }} / 2\left(=6.5 \mathrm{~mm}=0.257 \lambda_{0}\right)$. Other configuration parameters are kept constant, as described in Section II.

Fig. 2 shows the Poynting vectors observed in a plane parallel to the $x-y$ plane at $z=-B+\Delta z / 2$, where $\Delta z=$ $0.5 \mathrm{~mm}=0.02 \lambda_{0}$. This plane is very close to the plane in which the feed probe is located. Note that the Poynting vectors whose amplitude is less than $-25 \mathrm{~dB}$ are removed for simplicity. It is found that the parallel-plate mode power observed in the conventional $P$-type antenna is reduced in the newly proposed $C$-type antenna. The input power for the $C$-type antenna is concentrated in the aperture region.

The Poynting vectors in the $x-z$ plane are shown in Fig. 3. A comparison of Fig. 3(a) with (b) reveals that the cavity contributes to suppressing the generation of parallel-plate mode power between the top and bottom plates. For further information, the Poynting vectors in the $y-z$ plane are shown in Fig. 4. An asymmetrical power flow with respect to the $z$ axis is observed in free-space for the $P$-type antenna. On the other hand, an almost symmetrical power flow is observed for the $C$-type antenna.

The far-field radiation patterns resulting from the abovementioned power flow are shown in Fig. 5, where the measured radiation patterns are also presented. In this measurement, a large TTL (dimensions of the top and bottom plates are

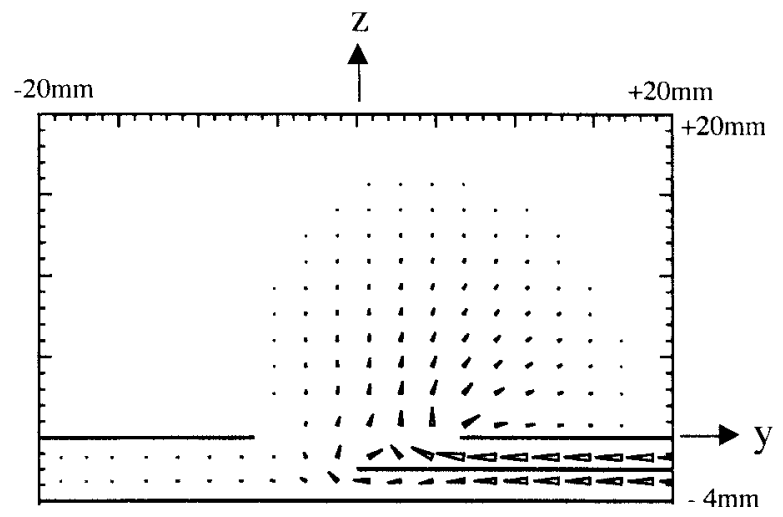

(a)

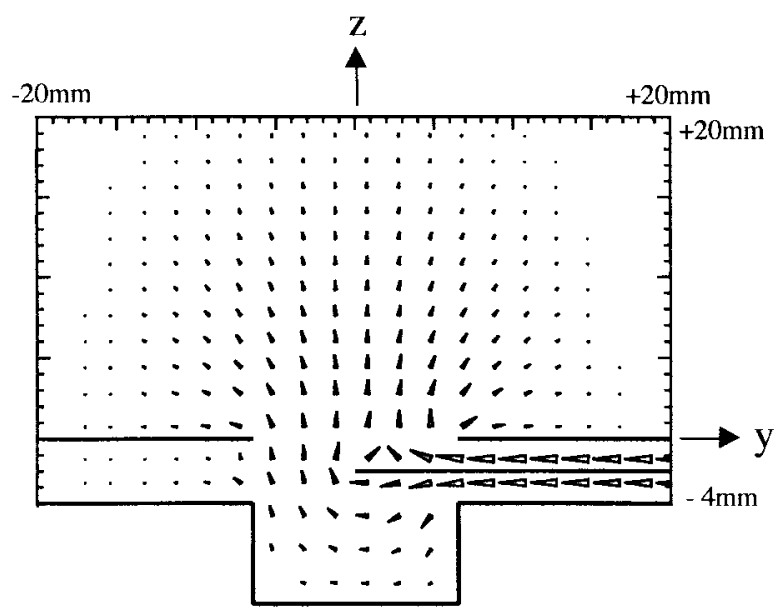

(b)

Fig. 4. Poynting vectors in the $y-z$ plane. (a) $P$-type antenna. (b) $C$-type antenna.

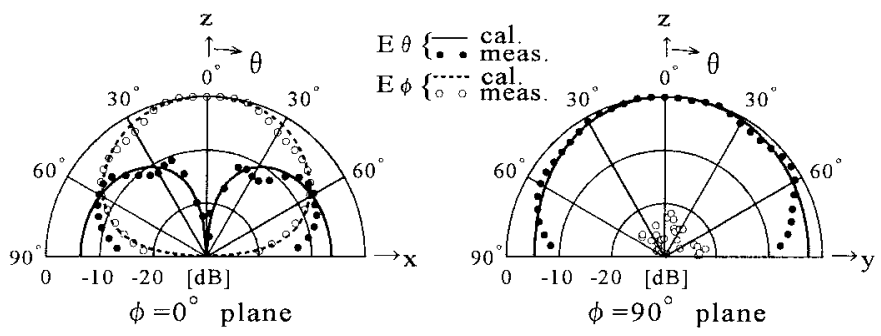

(a)

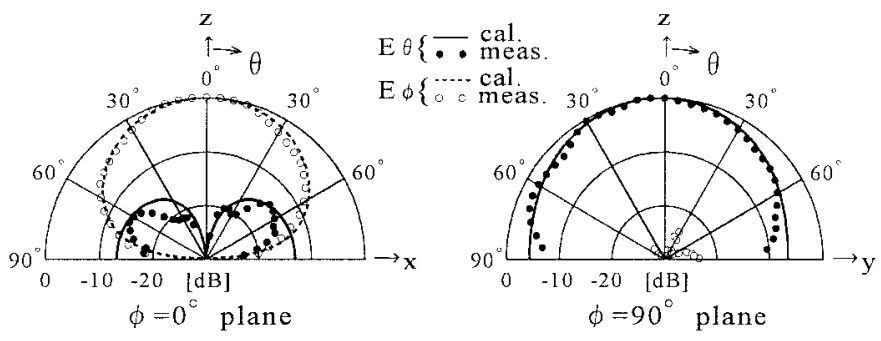

(b)

Fig. 5. Radiation patterns in the $\phi=0^{\circ}$ and $90^{\circ}$ planes. (a) $P$-type antenna. (b) $C$-type antenna.

$\left.x \times y=110 \mathrm{~mm} \times 110 \mathrm{~mm}=4.3 \lambda_{0} \times 4.3 \lambda_{0}\right)$ is used to approximate the analysis model i.e., the TTL having top and bottom plates of infinite extent. In addition, absorbing material 


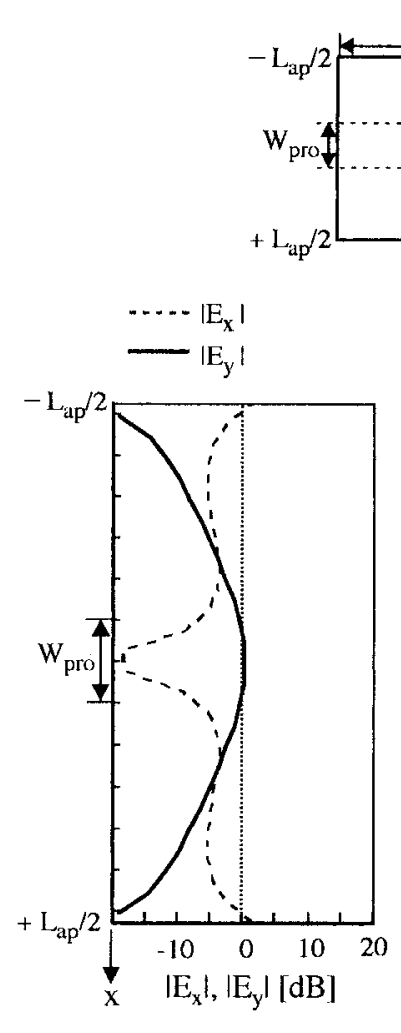

(a)

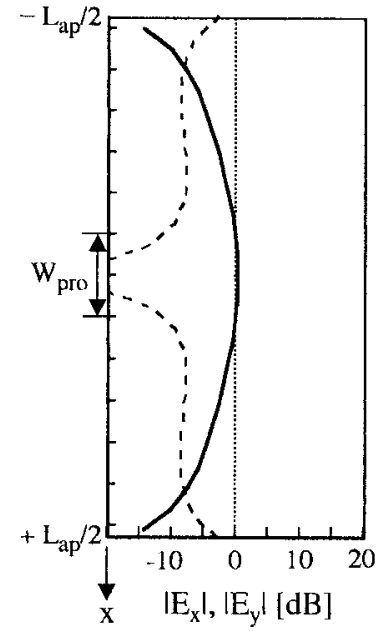

(b)
Fig. 6. Electric field components $E_{x}$ and $E_{y}$ on the $x$-axis at the aperture. (a) $P$-type antenna. (b) $C$-type antenna.

is attached to the inside of the vertical walls surrounding the TTL. It is seen that the measured radiation patterns agree with the calculated results except for angles near $\theta=90^{\circ}$. This disagreement can be attributed to the finite size of the measured antenna.

The copolarization component $E_{\phi}$ and cross-polarization component $E_{\theta}$ in the $x-z$ plane ( $\phi=0^{\circ}$ plane) are generated from the electric field components $E_{y}$ and $E_{x}$ at the aperture, respectively. Fig. 6(a) shows the amplitude values of $\left|E_{x}\right|$ and $\left|E_{y}\right|$ on the $x$ axis at the aperture of the $P$-type antenna and Fig. 6(b) shows those of the $C$-type antenna. The $C$-type antenna has a wider region of $\left|E_{y}\right|$ that satisfies $\left|E_{y}\right| \geq\left|E_{x}\right|$ than the $P$-type antenna. This implies that the effect of the $E_{x}$ component on the radiation pattern is smaller for the $C$-type antenna, as compared with that for the $P$-type antenna. In other words, the $C$-type antenna has a smaller cross polarization component than the $P$-type antenna, as shown in Fig. 5.

\section{B. Input Impedance and Radiation Efficiency as a Function of Probe Length}

The probe length $L_{\text {pro }}$ is kept constant in the above discussions: $L_{\text {pro }}=L_{\mathrm{ap}} / 2$. In this subsection, the radiation characteristics when the probe length $L_{\text {pro }}$ is changed are investigated.

Fig. 7 shows the input impedance $Z_{\text {in }}=R_{\text {in }}+j X_{\text {in }}$ as a function of relative probe length $L_{\text {pro }} / L_{\text {ap }}$. The input impedance is observed at the aperture edge $\left(y=L_{\mathrm{ap}} / 2\right)$. The variation of $Z_{\text {in }}$ for the $C$-type antenna is not as large as that

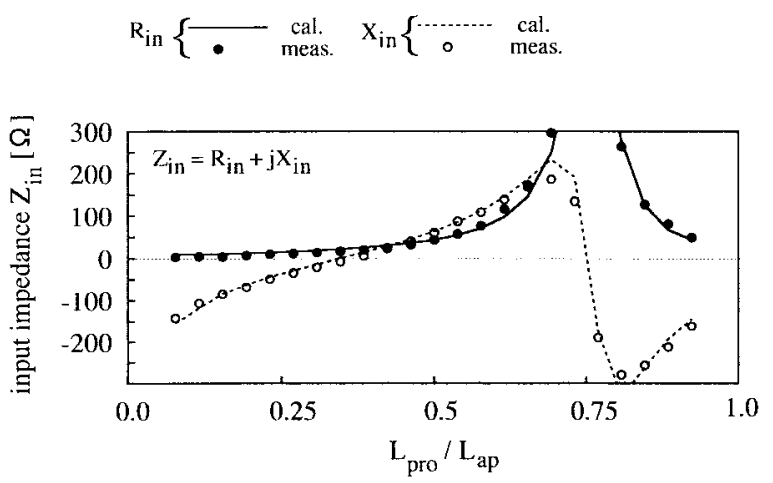

(a)

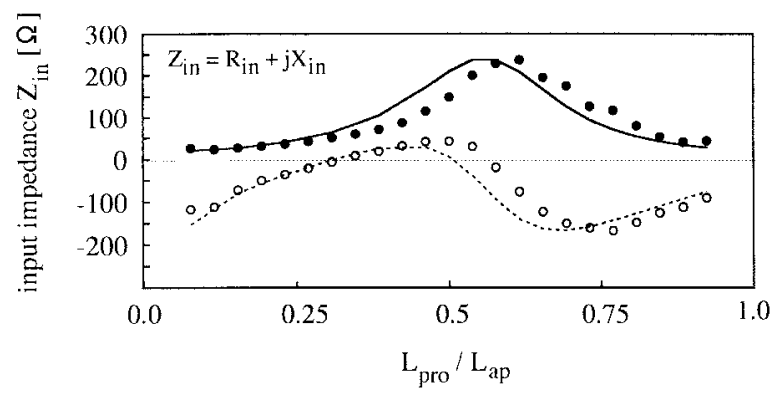

(b)

Fig. 7. Input impedance $Z_{\text {in }}=R_{\text {in }}+j X_{\text {in }}$ as a function of relative probe length $L_{\text {pro }} / L_{\text {ap }}$. (a) $P$-type antenna. (b) $C$-type antenna.

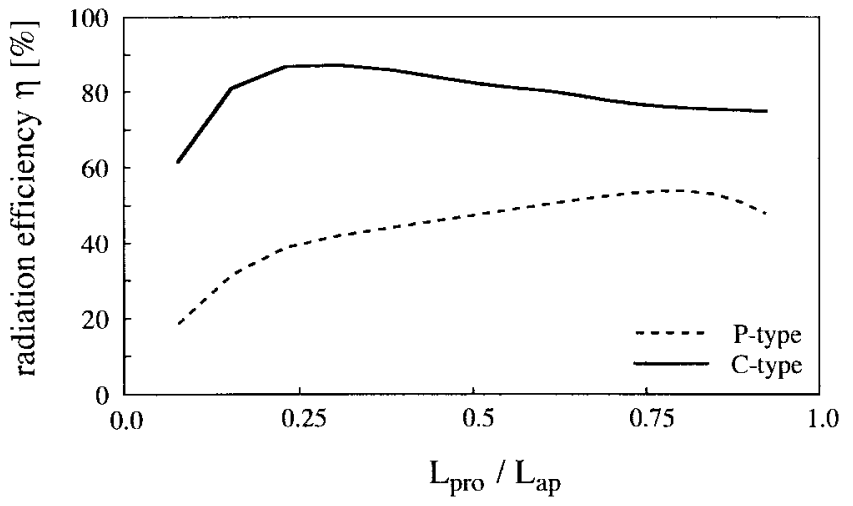

Fig. 8. Radiation efficiency $\eta$ as a function of relative probe length $L_{\text {pro }} / L_{\text {ap }}$.

for the $P$-type antenna. The $C$-type antenna has pure resistance values of approximately 65 and $210 \mathrm{ohms}$ at two probe lengths. The reactance values are very small over the range between these two probe lengths. The behavior of these resistance and reactance values simplifies the design for matching the feed line to the aperture.

The deterioration of radiation efficiency is due to the distribution of parallel-plate mode power in the TTL, which is a function of the probe length. Fig. 8 shows the radiation efficiency $\eta$ as a function of relative probe length $L_{\text {pro }} / L_{\text {ap }}$. It is found that the $C$-type antenna has higher radiation efficiency than the $P$-type antenna, as expected from comparison of the Poynting vectors shown in Figs. 2-4. At $L_{\text {pro }}=L_{\mathrm{ap}} / 2$, the radiation efficiency is $\eta=47 \%$ for the $P$-type antenna and $\eta=82 \%$ for the $C$-type antenna. It can be said that the 


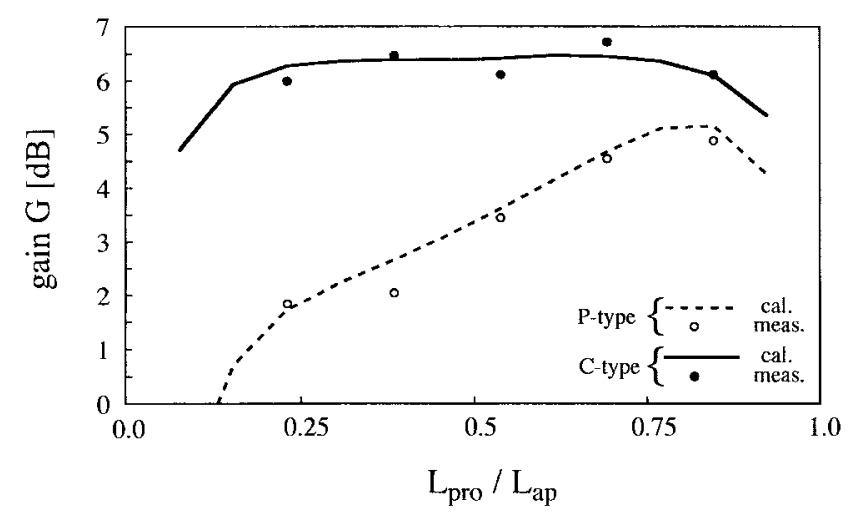

Fig. 9. Gain as a function of relative probe length $L_{\mathrm{pro}} / L_{\mathrm{ap}}$.

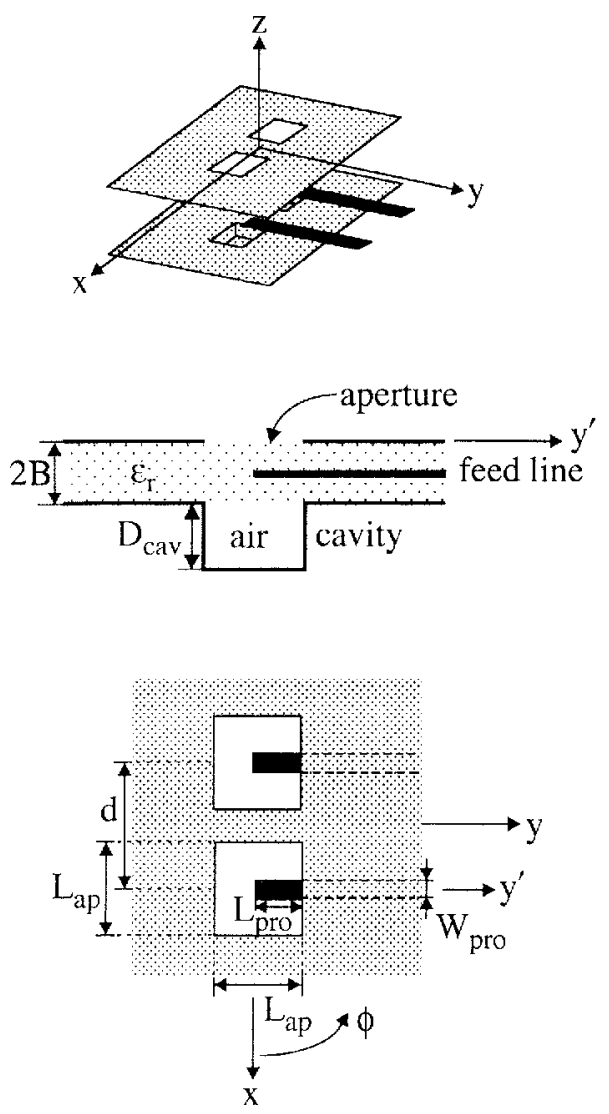

Fig. 10. Two-element array antenna.

$C$-type antenna significantly improves the radiation efficiency by virtue of reducing the parallel-plate mode power.

High radiation efficiency $\eta$ is essential for realizing high antenna gain $G$ ( $=\eta D$ with $D$ being directivity). Fig. 9 shows the gain $G$ when the probe length $L_{\text {pro }}$ is changed. It is clear that the $C$-type antenna has higher gain than the $P$ type antenna. It is also revealed that the gain of the $C$-type antenna is less sensitive to probe length, compared with that of the $P$-type antenna.

\section{Fundamental Two-Element Array Antenna}

It has been revealed that the newly proposed $C$-type antenna has better antenna characteristics than the conventional $P$ -
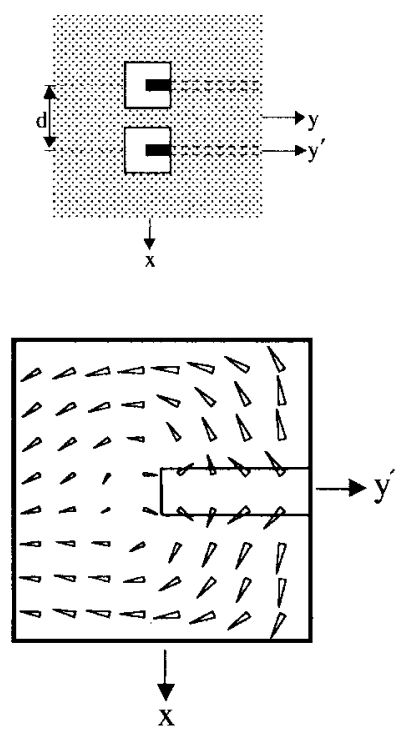

Fig. 11. Poynting vectors calculated for the aperture located on the $+x$ side (element spacing $d=0.8 \lambda_{0}$ ).

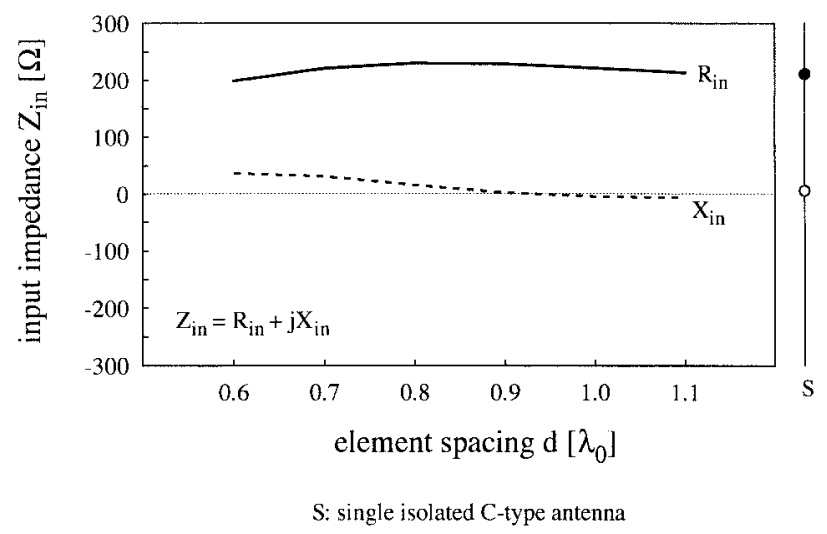

Fig. 12. Input impedance $Z_{\text {in }}$ of the two-element array antenna as a function of element spacing $d$.

type antenna. Further investigation, therefore, is focused on an application using the $C$-type antennas.

Fig. 10 shows a two-element array antenna, where the element spacing is designated as $d$. The configuration parameters $L_{\text {ap }}, W_{\text {pro }}, T_{\text {pro }}, \varepsilon_{r}, B$, and $D_{\text {cav }}$ for the array elements are the same as those described in Section II. The probe length is chosen to be $L_{\text {pro }}=L_{\text {ap }} / 2$.

The effects of the element spacing $d$ on the Poynting vectors in the aperture are small. A representative Poynting vector distribution is shown in Fig. 11. The Poynting vectors are calculated for the aperture located on the $+x$ side. Due to the cavity effects, the Poynting vector distribution remains almost symmetrical with respect to the $y^{\prime}$ axis (the feed line axis) for $d \geq 0.8 \lambda_{0}$ and is almost the same as that observed for a single isolated $C$-type antenna. This characteristic is used in the design of a much larger array antenna [6].

The input impedance $Z_{\text {in }}$ of the array antenna as a function of element spacing $d$ is presented in Fig. 12. The dots on the right side in this figure are the input impedance of a single isolated $C$-type antenna. It is seen that the variation in the input impedance with element spacing $d$ is not large over this 

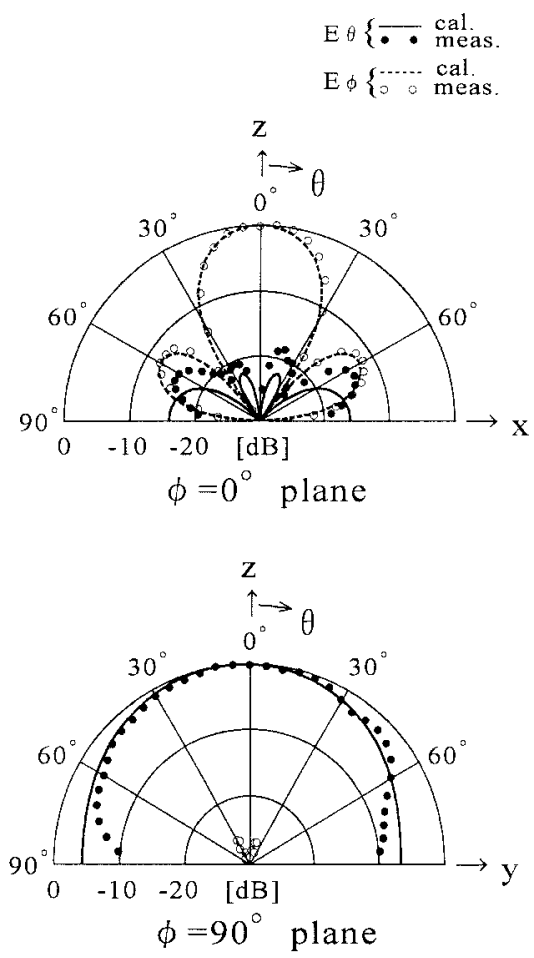

Fig. 13. Radiation patterns of the two-element array antenna in the $\phi=0^{\circ}$ and $90^{\circ}$ planes (element spacing $d=0.8 \lambda_{0}$ ).

analysis range. The input impedance for $d \geq 0.9 \lambda_{0}$ is almost the same as that for the single isolated $C$-type antenna.

Fig. 13 shows the theoretical radiation patterns together with measured data when the gain shows a maximum value of approximately $10 \mathrm{~dB}$ at $d=0.8 \lambda_{0}$. The theoretical and measured radiation patterns are in good agreement except for angles near $\theta=90^{\circ}$. The discrepancy near $\theta=90^{\circ}$ is due to the fact that the measurement is carried out using a TTL of finite extent, as in Fig. 5.

\section{CONCLUSION}

Conventional $P$ - and newly proposed $C$-type aperture antennas have been analyzed using the FDTD method. Each antenna is constructed using a TTL. The bottom of the $P$ type antenna is made of a planar conducting plate, while that of the $C$-type antenna is made of a conducting plate backed by a hollow cavity.

The Poynting vectors have been obtained for the two antennas. The undesirable parallel-plate mode power observed in the $P$-type antenna is lower in the $C$-type antenna. This means that the cavity is useful in reducing the parallel-plate mode power.

Investigation of the radiation pattern reveals that the $C$ type antenna has a smaller cross polarization component than the $P$-type antenna. This is interpreted from the electric field distribution over the aperture. It is also revealed that the input impedance for the $C$-type antenna is less sensitive to probe length than that for the $P$-type antenna. This simplifies the design for matching the feed line to the aperture. Further investigation shows that the $C$-type antenna has much higher radiation efficiency (more than $80 \%$ ) and gain than the $P$-type antenna due to reduction of the parallel-plate mode power.

Based on the above investigations, an analysis of the radiation characteristics of a fundamental array antenna composed of two $C$-type elements has been performed. The Poynting vector distributions in the apertures remain almost unchanged when the element spacing is varied. It is also found that the input impedance converges at an element spacing of approximately $0.9 \lambda_{0}$. The calculated radiation patterns agree with measured results.

\section{ACKNOWLEDGMENT}

The authors would like to thank V. Shkawrytko for his assistance in the preparation of this paper.

\section{REFERENCES}

[1] R. M. Sorbello, A. I. Zaghoul, J. E. Effland, and D. F. Difonzo, "A highefficiency flat plate array for direct broadcast satellite applications," in Proc. 18th Eur. Microwave Conf., Stockholm, Sweden, Sept. 1988, pp. 295-299.

[2] D. J. Sommers, "Slot array employing photoetched tri-plate transmission lines," IEEE Trans. Microwave Theory Tech., vol. MTT-3, pp. 157-162, Mar. 1955.

[3] N. K. Das, "Methods of suppression or avoidance of parallel-plate power leakage from conductor-backed transmission lines," IEEE Trans. Microwave Theory Tech., vol. 44, pp. 169-181, Feb. 1996.

[4] M. W. Shelley, D. J. Brain, A. Roederer, and K. Markus, "A novel high efficiency, dual polarized, flat plate array," in Proc. Int. Conf. Antennas Propagat (ICAP), Heriot-Watt University, Edinburgh, U.K., Mar. 1993, pp. 372-375.

[5] A. Taflove, Computational Electrodynamics: The Finite-Difference Time Domain Method. Norwood, MA: Artech House, 1995.

[6] K. Nakayama and H. Nakano, "Triplate-type aperture antenna backed by a cavity," Trans. IEICE, vol. J82-B, pp. 410-419, Mar. 1999.

[7] K. S. Yee, "Numerical solution of initial boundary value problems involving Maxwell's equations in isotropic media," IEEE Trans. Antennas Propagat., vol. AP-14, pp. 302-307, May 1966.

[8] Z. P. Liao, H. L. Wong, B. P. Yang, and Y. F. Yuan, "A transmitting boundary for transient wave analyses," Sci. Sinica, vol. 27, ser. A, no. 10, pp. 1063-1076, 1984.

[9] T. Uno, Finite Difference Time Domain Method for Electromagnetic Field and Antenna Analyses. Tokyo, Japan: Corona, 1998, pp. 172-173.

[10] M. De Pourcq, "Field and power-density calculations in closed microwave systems by three-dimensional finite differences," Proc. Inst. Elect. Eng., vol. 132, pt. H, pp. 360-368, Oct. 1985.

[11] R. F. Harrington, Time-Harmonic Electromagnetic Fields. New York McGraw-Hill, 1961, pp. 106-110.

[12] C. A. Balanis, Antenna Theory. New York: Harper Row, 1982, ch. 11.

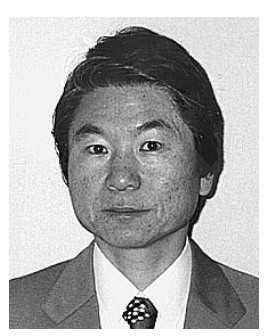

Hisamatsu Nakano (M'75-SM'87-F'92) received the B.E., M.E., and Dr.E. degrees in electrical engineering from Hosei University, Tokyo, in 1968, 1970, and 1974, respectively.

Since 1973, he has been a member of the faculty of Hosei University, where he is now a Professor of Electronic Informatics Department. He was a Visiting Associate Professor at Syracuse University, Syracuse, NY (May to September 1981), a Visiting Professor at University of Manitoba Canada (March to September 1986), and a Visiting Professor at University of California, Los Angeles (September 1986 to March 1987). He has published more than 150 refereed journal papers on antenna and lightwave problems.

Dr. Nakano received the IEEE AP-S Best Application Paper Award (H. A. Wheeler Award) in 1994. He is an Associate Editor of the IEEE Antennas and Propagation Magazine. 


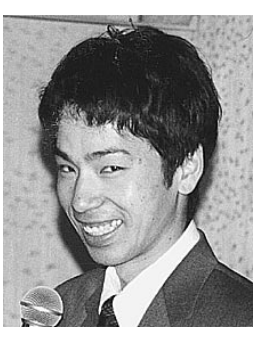

Tomohiro Unno was born in Shizuoka, Japan, on January 9, 1974. He received the B.E. and M.E. degrees in electrical engineering from Hosei University, Tokyo, in 1996 and 1998, respectively.

Since 1998, he has been with Digital Press Corporation, Tokyo, Japan. His main interests are in the design and analysis of mobile communication antennas.

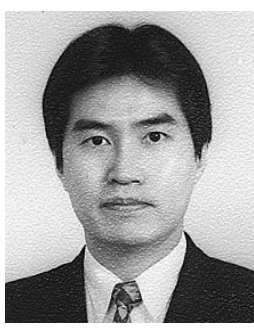

Kazuo Nakayama (M'98) was born in Hiroshima, Japan, on January 20, 1966. He received the B.E., M.E., and Dr.E. degrees in electrical engineering from Hosei University, Tokyo, Japan, in 1989, 1991, and 1999 , respectively.

From 1991 to 1994, he was with Uniden Corporation, Chiba, Japan. His current research interests are in the design and analysis of print antennas for mobile and satellite communication systems.

Dr. Nakayama is a member of the Institute of Electronics, Information, and Communication Engi-

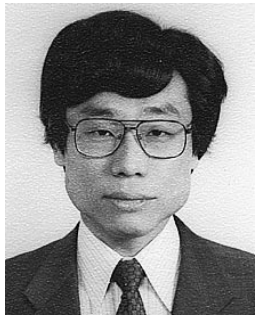

Junji Yamauchi (M'85) was born in Nagoya, Japan, on August 23, 1953. He received the B.E., M.E., and Dr.E. degrees from Hosei University, Tokyo, Japan, in 1976, 1978, and 1982, respectively.

From 1984 to 1988, he served as a Lecturer in the Electrical Engineering Department of Tokyo Metropolitan Technical College, Japan. Since 1988 he has been a member of the faculty of Hosei University, where he is now a Professor of Electronic Informatics Department. His research interests include optical waveguides and circularly polarized antennas.

Dr. Yamauchi is a member of the Optical Society of America and the Institute of Electronics, Information and Communication Engineers of Japan.

neers (IEICE) of Japan. 